\title{
DIFERENCIAÇÃO POR ORIGEM E SUBSTITUIÇÃO NAS EXPORTAÇÕES DE SOJA EM GRÃO DO BRASIL, DOS EUA E DA ARGENTINA ${ }^{1}$
}

\author{
Adelson Martins Figueiredo ${ }^{2}$ \\ Maurinho Luiz dos Santos ${ }^{3}$ \\ Viviani Silva Lirio ${ }^{4}$
}

Resumo - O objetivo deste trabalho foi estimar e analisar a elasticidade de substituição entre Brasil, EUA e Argentina nas exportações de soja em grão, em mercados importadores selecionados. Esse fato é importante, na medida em que aumenta a integração das economias mundiais, colocando em destaque a adaptabilidade às exigências da demanda mundial, como fator fundamental para expansão das exportações. Assim, dada a relevância econômica das exportações brasileiras de soja em grão, buscou-se, especificamente, verificar a possibilidade de substituição entre as exportações de soja em grão do Brasil, dos EUA e da Argentina. Após realização de testes Dickey-Fuller expandido $(\mathrm{ADF})$, para estacionariedade e testes traço e de máximo autovalor para co-integração, adotou-se o Modelo de Correção de Erros (MCE) na estimativa da elasticidade de substituição. Esse modelo mostrou-se bem ajustado aos dados e permitiu identificar a presença ou ausência de rigidez de mercado, ademais, que não há substitutibilidade perfeita nas exportações de soja em grão. Dessa maneira, investimento em diferenciação do produto, via rastreabilidade e preservação de identidade, pode impulsionar as exportações brasileiras.

Palavras-chave: Elasticidade de substituição, exportações, soja, Brasil.

\footnotetext{
${ }^{1}$ Recebido em 05/09/2004

Aceito em 29/10/2004

${ }^{2}$ Doutorando em Economia Aplicada pelo Departamento de Economia Rural da UFV e bolsista da CAPES, email: martinsfigueiredo@yahoo.com.br.

3 Professor do Departamento de Economia Rural da UFV, e-mail: mlsantos@ufv.br.

${ }^{4}$ Professora do Departamento de Economia Rural da UFV, e-mail: vslirio@ufv.br
} 


\section{Introdução}

Na década de 1990, a agroindústria da soja teve participação média de $8,6 \%$ no valor das exportações brasileiras totais. Em 2003, as exportações dessa agroindústria foram de US\$ 8,12 bilhões, sendo o segmento de soja em grão responsável por 52,8\% (MDIC, 2004). Diante dessas circunstâncias, a exportação de soja em grão torna-se fundamental na geração de divisas para o Brasil.

Essas considerações são importantes, pois a tomada de decisões tornase questão essencial para expansão das exportações de soja em grão, principalmente porque esse mercado é caracterizado por pressões competitivas e constante disputa por novos mercados entre Argentina (ARG), Brasil (BRA) e Estados Unidos (EUA). De acordo com dados da Food and Agriculture Organization (FAO), a participação desses três países foi de, aproximadamente, $82 \%$ no total da produção mundial em 2002 , e no valor das exportações mundiais totais obtiveram, respectivamente, as proporções de $10,6 \%, 27,7 \%$ e $53,3 \%$ da soja em grão comercializada no mercado mundial.

Do lado da demanda também existem grandes compradores, sobressaindo como principais importadores de soja em grão a China, Países Baixos, Japão, México, Alemanha e Espanha, os quais importam $64 \%$ do total de grãos de soja vendidos no mercado internacional (FAO, 2004). Essa estrutura do mercado mundial de soja em grão evidencia que, para uma análise mais segura dos fluxos comerciais desse produto, é necessário conhecer também os determinantes da demanda. Isso ocorre porque, ainda que o produto analisado seja uma commodity, existem características que são específicas da demanda de cada país importador. Fontes et al. (1989), ao analisarem as exportações de grão dos EUA, salientaram que funções de demandas diferenciadas por origem e destino fornecem informações importantes sobre os concorrentes, principalmente quando há competição no mercado analisado. Além desse trabalho, Viana et al. (2003) estudaram também a diferenciação por origem na demanda externa de cafés. 
As especificidades da demanda de soja em grão de cada país importador estão comumente ligadas, de forma direta, ao destino que será dado a essa commodity no mercado interno - isto é, se vão ser destinadas ao consumo imediato ou às indústrias de processamento - e, de forma indireta, às peculiaridades culturais que afetam os gostos e preferências dos consumidores, aos diferentes níveis de renda, aos variados níveis de preços praticados pelos exportadores, às imperfeições de mercado e aos custos de transação. Assim, os produtores que melhor adaptarem seus produtos às normas institucionais, culturais e sanitárias dos importadores conquistarão maiores parcelas desses mercados.

Além disso, a difusão do processo de integração entre os países, conjuntamente ao movimento de maior diversificação das pautas de exportações, colocou em destaque a adaptabilidade às exigências da demanda mundial como fator determinante do acompanhamento da dinâmica competitiva no mercado internacional. Nesse contexto, o que se propõe nesta pesquisa é utilizar uma metodologia simplificada para estimar a elasticidade de substituição nas exportações de soja em grão do Brasil (BRA), dos Estados Unidos (EUA) e da Argentina (ARG) para os seguintes mercados importadores: Alemanha, Espanha, França, Japão e Países Baixos. Especificamente, pretende-se verificar a possibilidade de substituição das exportações de soja em grão do Brasil, EUA e Argentina.

\section{Metodologia}

\subsection{Referencial teórico}

Os modelos de elasticidade de substituição (ES) pressupõem que os produtos sejam não-homogêneos, conforme Armington (1969a, b). Com base nessa pressuposição, considera-se que os consumidores sejam capazes de diferenciar produtos devido à sua localização geográfica de origem (por exemplo, soja brasileira versus soja argentina). Assim, se um produto originado ou produzido por diferentes regiões possui diferenças na visão do consumidor, a pressuposição de substitutibilidade perfeita não 
deve ser sustentável, e o modelo de demanda para aquele bem deve considerar que cada região seja ofertante de um produto diferente.

A elasticidade de substituição entre produtos agrícolas exportados por dois países competidores no mercado internacional é definida a partir do processo de maximização da utilidade com restrição orçamentária, pela seguinte expressão:

$$
e_{s}=\frac{\partial\left(q_{1} / q_{2}\right)}{\partial\left(\partial q_{1} / \partial q_{2}\right)} \times \frac{\partial q_{1} / \partial q_{2}}{q_{1} / q_{2}}=\frac{\partial \log \left(q_{1} / q_{2}\right)}{\partial \log \left(q_{2} / q_{1}\right)} \text {. }
$$

A equação (1) indica que a elasticidade de substituição entre dois produtos ou mercadorias é medida pela taxa de variação percentual, na razão das quantidades demandadas $\left(q_{1} / q_{2}\right)$, por meio da variação percentual da taxa marginal de substituição de $q_{2}$ por $q_{1}$.

De acordo com Fontes et al. (1989), esse conceito da elasticidade de substituição permite que esta seja derivada diretamente da curva de indiferença do consumidor. Dessa maneira, a elasticidade de substituição mede a capacidade de um produto exportado por um país substituir um produto similar exportado por outro país concorrente ao longo da curva de indiferença da nação importadora, sem alterar seu nível de satisfação. Essa abordagem é comumente usada em estudos que pressupõem algum grau de diferenciação do produto com origem em diferentes competidores no comércio internacional. De acordo com Binger e Hoffman (1998) e Varian (1999), esse processo pode ser obtido do seguinte problema de maximização:

$$
\operatorname{Max} \mathrm{U}\left(\mathrm{q}_{1}, q_{2}\right) \text { sujeito a } R=p_{1} q_{1}+p_{2} q_{2} \text {. }
$$

Considerando que a equação (2) seja representativa do processo de maximização da utilidade de uma nação importadora, em que $q_{1}$ e $q_{2}$ 
são quantidades consumidas de dois produtos similares, $p_{1}$ e $p_{2}$ são preços de exportação desses produtos do país A e de seu concorrente no comércio internacional, pode-se mostrar, pelas condições de primeira ordem, que:

$$
\frac{\partial q_{2}}{\partial q_{1}}=\frac{p_{1}}{p_{2}}
$$

Assim, ao substituir a equação (3) pela equação (1), obtém-se a formulação empírica da elasticidade de substituição, em que:

$$
e_{s}=\frac{\partial\left(q_{1} / q_{2}\right)}{\partial\left(\partial q_{1} / \partial q_{2}\right)} \times \frac{\partial q_{1} / \partial q_{2}}{q_{1} / q_{2}}=\frac{\partial \log \left(q_{1} / q_{2}\right)}{\partial \log \left(p_{1} / p_{2}\right)} \text {. }
$$

Rearranjando os termos da equação (4), é possível obter, de forma direta, a elasticidade de substituição $\left(e_{s}\right)$ :

$$
\log \left(q_{1} / q_{2}\right)=a+e_{s} \log \left(p_{1} / p_{2}\right)
$$

Quando a elasticidade de substituição $\left(e_{s}\right)$, obtida pela equação (5), for de sinal negativo e exceder a unidade em valores absolutos, significa que os produtos produzidos pelo país A e por seu concorrente são considerados substitutos próximos pelos mercados importadores. Um valor baixo da elasticidade de substituição, ou seja, $\left(-1<e_{s}<1\right)$, demonstra que o grau de competição entre os produtos 1 e 2 é pequeno. Segundo Leamer e Stern (1970), uma elasticidade de substituição baixa demonstra que os produtos são provavelmente diferenciados por país de origem, devido a vários fatores, como diferenças culturais que afetam as preferências dos países consumidores; acordos institucionais; variados níveis de renda; preços praticados pelos exportadores; imperfeições de mercado; e custos de transação. 
A definição teórica da $e_{s}$, com relação a uma única curva de indiferença, gera problemas de estimação em trabalhos empíricos. Todavia, Leamer e Stern (1970) afirmaram que a condição necessária para que a elasticidade de substituição esteja relacionada apenas com a razão dos preços é de que a inclinação da curva de indiferença que a originou esteja relacionada apenas com $\left(q_{1} / q_{2}\right)$, sendo indiferente a outros fatores relacionados com a renda.

Para que a equação (5) apresente resultados consistentes, teoricamente, as seguintes condições de simetria devem se verificar: a) A soma algébrica das elasticidades-preço direta e cruzada da demanda seja igual dos dois bens; e b) As elasticidades-preço, bem como as elasticidades-renda, da demanda para os dois bens sejam iguais (Silva, 1992). Caso contrário, as estimativas obtidas para a elasticidade de substituição podem diferir do conceito teórico, resultando em coeficientes $e_{s}>0$, que mostra complementaridade, e não substitutibilidade.

\subsection{Referencial analítico}

O procedimento econométrico para estimar a elasticidade de substituição entre a soja em grão do Brasil, dos EUA e da Argentina foi o Modelo de Correção de Erros (MCE). Esse modelo é comumente utilizado pelo fato de as séries de comércio internacional serem não-estacionárias; assim, o que geralmente ocorre é a regressão de uma série temporal não-estacionária sobre outra também não-estacionária, gerando parâmetros estimados não-confiáveis. Bacchi et al. (2002) e Figueiredo e Silva (2004), ao usarem dados mensais e anuais, respectivamente, para a cadeia brasileira de soja e para o segmento de soja em grão, constataram que as séries temporais das exportações brasileiras de soja e derivados são não-estacionárias em nível.

Portanto, da equação (5) pode-se montar o seguinte modelo estatístico que permite estimar a elasticidade de substituição entre o Brasil e con- 
correntes no mercado internacional de soja em grão, farelo e óleo produtos que serão denotados pelo símbolo $(i)$ :

$$
\log \left(q_{i j}^{B} / q_{i j}^{S}\right)_{t}=\beta_{0}+\beta_{1} \log \left(p_{i j}^{B} / p_{i j}^{S}\right)_{t}+\varepsilon_{t},
$$

em que $q_{i j}^{B}$ é quantidade exportada, pelo Brasil $(B)$, do produto $(i)$ para o país de destino $(j)$, em toneladas; $q_{i j}^{S}$, quantidade exportada, pelo concorrente $(s)$, do produto $(i)$ para o país de destino $(j)$, em toneladas; $p_{i j}^{B}$, preço de exportação praticado pelo $\operatorname{Brasil}(B)$ no mercado de destino $(j)$, em US\$ FOB por tonelada do produto $(i) ; p_{i j}^{S}$, preço de exportação praticado pelo concorrente $(s)$ no mercado de destino $(j)$, em US\$ FOB por tonelada do produto $(i) ; \boldsymbol{E}_{t}$, termo de erro aleatório com distribuição normal, isto é, média zero e variância constante; e $t$, período de tempo, medido, nesse caso, em semestres. O coeficiente $\beta_{1}$ representa a elasticidade de substituição, indicada como $\left(e_{s}\right)$, pela equação (5). De acordo com as relações entre as variáveis, espera-se que $\beta_{1}>|1|$ e que $\beta_{0}$, por ser o intercepto da função, possam variar de sinal.

Outro procedimento comum é estimar a elasticidade de substituição com a variável dependente defasada como variável explicativa. Fontes et al. (1989) e Stalder (1997) salientaram que a inclusão dessa variável é importante porque permite analisar a rigidez de mercado, decorrente da intervenção governamental no setor. Neste trabalho serão estimados os dois modelos - sem e com a variável dependente defasada como variável explicativa; dessa maneira, o modelo que inclui a rigidez de mercado $(\varphi)$ tem a seguinte forma funcional: 


$$
\log \left(q_{i j}^{B} / q_{i j}^{S}\right)_{t}=\beta_{0}+\beta_{1} \log \left(p_{i j}^{B} / p_{i j}^{S}\right)_{t}+\varphi_{2} \log \left(q_{i j}^{B} / q_{i j}^{S}\right)_{t-1}+\varepsilon_{t} .
$$

Segundo Griffiths et al. (2000), o MCE é estimado em um Processo de Diferença Estacionária (PDE). Então, a partir da equação (6), pode-se ilustrar esse mecanismo de diferenciação da seguinte forma:

$$
\Delta \log \left(q_{i j}^{B} / q_{i j}^{S}\right)_{t}=\log \left(q_{i j}^{B} / q_{i j}^{S}\right)_{t}-\log \left(q_{i j}^{B} / q_{i j}^{S}\right)_{t-1} .
$$

Tomando a primeira defasagem da equação (6) e substituindo-a em (8), é possível identificar que:

$$
\Delta \log \left(q_{i j}^{B} / q_{i j}^{S}\right)_{t}=\alpha_{0}+\alpha_{1} \Delta \log \left(p_{i j}^{B} / p_{i j}^{S}\right)_{t}+\Delta \varepsilon_{t} .
$$

Ao estimar a equação (9) em diferença, possivelmente perdem-se as informações de longo prazo que seriam obtidas pela equação (6). O MCE consiste em corrigir esse problema, incluindo o erro defasado $\left(\hat{\varepsilon}_{t-1}\right)$ estimado, obtido por Mínimos Quadrados Ordinários (MQO) da equação (6), na equação (9), como segue:

$$
\Delta \log \left(q_{i j}^{B} / q_{i j}^{S}\right)_{t}=\alpha_{0}+\alpha_{1} \Delta_{1} \log \left(p_{i j}^{B} / p_{i j}^{S}\right)_{t}+\omega\left(\hat{\varepsilon}_{t-1}\right)+\mu_{t},
$$

em que $\Delta$ é o operador de primeira diferença; $\left(\hat{\varepsilon}_{t-1}\right)$, termo de erro da equação (6), que é defasado em um período e representa $\Delta \varepsilon_{t}$ da equação (9); e $(\omega)$, parâmetro equilibrador de longo prazo. Esse procedimento é válido também para a equação (7). 
Para otimizar os resultados obtidos pela aplicação do MCE, as séries temporais utilizadas devem ser integradas de mesma ordem e co-integradas. Dessa forma, realizou-se o teste de identificação de raiz unitária, que definiu se as séries estudadas eram estacionárias, ou não, e qual a ordem de integração destas, permitindo a realização do teste de cointegração entre as variáveis ${ }^{5}$.

\subsubsection{Teste de autocorrelação}

Segundo Gujarati (2000), em modelos em que se inclui a variável dependente defasada como variável explicativa, como na equação (7), o teste de Durbin-Watson torna-se inadequado para detectar a presença de autocorrelação. Nesse caso, pode-se detectar a presença de autocorrelação por meio do modelo auto-regressivo de heteroscedasticidade condicional $(\mathrm{ARCH})$. Esse modelo pressupõe que a variância do erro aleatório $\mu_{t}$ dependa do tamanho do termo de erro passado, elevado ao quadrado.

O processo $\mathrm{ARCH}(p)$ pode ser escrito da seguinte forma:

$$
\operatorname{Var}\left(\mu_{t}\right)=\alpha_{t}^{2}=\alpha_{0}+\alpha_{t} \mu_{t-1}^{2}+\alpha_{t} \mu_{t-2}^{2}+\ldots+\alpha_{p} \mu_{t-p}^{2}
$$

Se não houver autocorrelação na variância do erro, ter-se-á $H_{0}=\alpha_{1}=\alpha_{2}=\ldots=\alpha_{p}=0$, o que implica que $\operatorname{var}\left(\mu_{t}\right)=\alpha_{0}$ é homoscedástica. $\mathrm{H}_{0}$ pode ser testada por meio do teste $\mathrm{F}$ de uma regressão estimada com $\mu_{\mathrm{t}}$ (erro estimado obtido da equação (10)) ou pelo coeficiente $n \cdot R^{2}$, que segue distribuição qui-quadrado $\left(\chi^{2}\right)$, sendo $n o$ número de observações e $R^{2}$, coeficiente de determinação obtido da equação (11).

\footnotetext{
${ }^{5}$ A formulação matemática, bem como os resultados destes testes, pode ser consultada em Figueiredo (2004).
} 


\subsubsection{Fonte de dados e variáveis}

Os dados utilizados nesta pesquisa foram obtidos nas seguintes instituições: a) Food and Agriculture Organization (FAO); b) United States Department of Agriculture (USDA), por meio do Economic Research Service (ERS); c) Ministério da Agricultura, Indústria e Comércio Exterior (MDIC), mediante Secretaria de Comércio Exterior (SECEX); e d) Ministerio de Economía y Producción de la Republica Argentina (MECON), no Instituto Nacional de Estadística y Censos (INDEC).

Para estimativa da elasticidade de substituição entre Brasil, Argentina e EUA, os dados são semestrais e compreendem o período de janeiro de 1990 a julho de 2003. As séries de quantidade exportada de soja em grão, em toneladas métricas, e o valor em US\$1000,00 Free on Board (FOB) do Brasil, para cada país de destino selecionado e apresentado no item seguinte, foram obtidos do MDIC/SECEX. Para a Argentina, essas mesmas séries foram cedidas pelo INDEC/MECON, e as séries referentes às exportações dos EUA foram obtidas no USDA/ERS. Para obtenção das séries de preços de exportação e de importação referentes a cada país de destino selecionado, dividiu-se o valor das vendas totais pelas respectivas quantidades transacionadas de cada exportador, em cada mercado importador.

\section{Resultados e discussão}

\subsection{Elasticidade de substituição e rigidez de mercado}

Depois da aplicação dos testes de estacionariedade e co-integração, procedeu-se à estimação do Modelo de Correção de Erros (MCE), que foi usado para mensurar a rigidez de mercado e a elasticidade de substituição entre Brasil, EUA e Argentina nas exportações de soja e derivados para mercados importadores distintos, de acordo com a adequação e a disponibilidade de dados. 
Esse procedimento é importante porque, além de evitar a ocorrência de regressão espúria, ameniza o efeito da sazonalidade sobre os parâmetros da elasticidade de substituição ${ }^{6}$, uma vez que a aplicação do MCE é realizada sobre as séries em diferença. Os resultados do modelo são apresentados na Tabela 1. Nesta tabela constam também os testes DW para o modelo 1, que representa a equação (6) sem variáveis defasadas, e o teste ARCH para o modelo 2, com variáveis explicativas defasadas (equação 7). A autocorrelação não foi detectada em todas as regressões, conforme estimativas desses testes.

Os parâmetros equilibradores de longo prazo, incluídos nos modelos $1 \mathrm{e}$ 2 , foram negativos e significativos. Assim, a discrepância entre os valores das elasticidades, no curto e no longo prazo, será corrigida na proporção de cada um desses parâmetros de equilíbrio para cada mercado importador, a cada semestre. $\mathrm{O}$ sinal negativo significa que, se esses parâmetros não estivessem contidos nos modelos, as elasticidades de longo prazo seriam superestimadas.

Na Tabela 1 são apresentadas as respostas de preço das exportações de soja em grão, quando o Brasil compete com os EUA e com a Argentina. Os resultados confirmam a hipótese de relação inversa entre a razão das quantidades exportadas do Brasil versus competidores e seus relativos preços de exportação.

\footnotetext{
${ }^{6}$ Neste estudo, esse procedimento torna-se relevante devido à complementaridade entre a safra sul-americana e a norte-americana, ou seja, nos Estados Unidos colhe-se a partir de setembro e o pico da comercialização vai até dezembro, enquanto no Cone Sul a colheita inicia-se em março e a comercialização vai até junho.
} 
Tabela 1 - Estimativa da elasticidade de substituição entre o Brasil e seus principais concorrentes nas exportações mundiais de soja em grão, por mercado importador, no período de 1990 a 2003

\begin{tabular}{|c|c|c|c|c|c|c|}
\hline Exportadores - Importador & Var. & $\begin{array}{c}\text { Modelo } \\
1\end{array}$ & "t" stat. & Var. & $\begin{array}{c}\text { Modelo } \\
2^{(1)}\end{array}$ & "t" stat. \\
\hline \multirow{6}{*}{ BRA / EUA - Alemanha } & Cte & $0,06^{(\mathrm{ns})}$ & 0,4536 & Cte & $-0,02^{(\mathrm{ns})}$ & $-0,2139$ \\
\hline & $\left(e_{\mathrm{s}}\right)$ & $-4,45^{(*)}$ & $-3,0331$ & $\left(e_{\mathrm{s}}\right)$ & $-0,52^{(\mathrm{ns})}$ & $-0,5596$ \\
\hline & $(\omega)$ & $-0,24^{(* * *)}$ & $-1,8314$ & $(\varphi)$ & $1,06^{(*)}$ & 7,5927 \\
\hline & $\left(\mathrm{R}^{2}\right)$ & 0,3440 & & $(\omega)$ & $-1,28^{(*)}$ & $-5,5288$ \\
\hline & DW & 2,3987 & & $\left(\mathrm{R}^{2}\right)$ & 0,8169 & \\
\hline & & & & $\mathrm{ARCH}$ & 0,6026 & $0,437^{\text {(p.v) }}$ \\
\hline \multirow{6}{*}{ BRA / ARG - Espanha } & Cte & $0,07^{(\mathrm{ns})}$ & 0,3292 & Cte & $0,03^{\text {(ns) }}$ & 0,1180 \\
\hline & $\left(e_{\mathrm{s}}\right)$ & $-5,75^{(*)}$ & $-7,8425$ & $\left(e_{\mathrm{s}}\right)$ & $-6,09^{(*)}$ & $-7,3140$ \\
\hline & $(\omega)$ & $-0,56^{(*)}$ & $-2,7321$ & $(\varphi)$ & $0,13^{(\mathrm{ns})}$ & 1,2321 \\
\hline & $\left(\mathrm{R}^{2}\right)$ & 0,7304 & & $(\omega)$ & $-0,69^{(*)}$ & $-2,6010$ \\
\hline & DW & 1,6566 & & $\left(\mathrm{R}^{2}\right)$ & 0,7324 & \\
\hline & & & & $\mathrm{ARCH}$ & 0,7810 & $0,676^{\text {(p.v) }}$ \\
\hline \multirow[t]{6}{*}{ BRA / EUA - Espanha } & Cte & $0,03^{\text {(ns) }}$ & 0,3151 & Cte & $-0,03^{(\mathrm{ns})}$ & $-0,5876$ \\
\hline & $\left(e_{\mathrm{s}}\right)$ & $-7,35^{(*)}$ & $-6,8283$ & $\left(e_{\mathrm{s}}\right)$ & $-5,12^{(*)}$ & $-8,3485$ \\
\hline & $(\omega)$ & $-0,83^{(*)}$ & $-4,2117$ & $(\varphi)$ & $0,68^{(*)}$ & 9,4765 \\
\hline & $\left(\mathrm{R}^{2}\right)$ & 0,7247 & & $(\omega)$ & $-1,22^{(*)}$ & $-6,6546$ \\
\hline & DW & 1,8620 & & $\left(\mathrm{R}^{2}\right)^{\prime}$ & 0,9288 & \\
\hline & & & & $\mathrm{ARCH}$ & 0,1734 & $0,926^{\text {(p.v) }}$ \\
\hline \multirow{6}{*}{ BRA / EUA - França } & Cte & $0,08^{(\mathrm{ns})}$ & 0,3413 & Cte & $-0,11^{(\mathrm{ns})}$ & $-0,9499$ \\
\hline & $\left(e_{\mathrm{s}}\right)$ & $-4,02^{(* * *)}$ & $-1,8770$ & $\left(e_{\mathrm{s}}\right)$ & $-2,47^{(* *)}$ & $-2,2882$ \\
\hline & $(\omega)$ & $-0,96^{(*)}$ & $-4,7572$ & $(\varphi)$ & $1,16^{(*)}$ & 8,1336 \\
\hline & $\left(\mathrm{R}^{2}\right)$ & 0,5261 & & $(\omega)$ & $-1,49^{(*)}$ & $-9,1118$ \\
\hline & DW & 1,3663 & & $\left(\mathrm{R}^{2}\right)$ & 0,8460 & \\
\hline & & & & $\mathrm{ARCH}$ & 3,5080 & $0,173^{(\mathrm{p} . \mathrm{v})}$ \\
\hline \multirow[t]{6}{*}{ BRA / EUA - Japão } & Cte & $-0,01^{(\mathrm{ns})}$ & $-0,0800$ & Cte & $-0,01^{\text {(ns) }}$ & $-0,3146$ \\
\hline & $\left(e_{\mathrm{s}}\right)$ & $-0,43^{(\mathrm{ns})}$ & $-0,3197$ & $\left(e_{\mathrm{s}}\right)$ & $0,39^{(* *)}$ & 2,1093 \\
\hline & $(\omega)$ & $-0,98^{(*)}$ & $-4,7937$ & $(\varphi)$ & $1,07^{(*)}$ & 47,937 \\
\hline & $\left(\mathrm{R}^{2}\right)$ & 0,5003 & & $(\omega)$ & $-1,39^{(*)}$ & $-7,3586$ \\
\hline & DW & 1,9883 & & $\left(\mathrm{R}^{2}\right)$ & 0,9909 & \\
\hline & & & & $\mathrm{ARCH}$ & 1,7085 & $0,425^{\text {(p.v) }}$ \\
\hline \multirow[t]{6}{*}{ BRA / ARG - Países Baixos } & Cte & $0,13^{\text {(ns) }}$ & 0,6323 & Cte & $0,05^{\text {(ns) }}$ & 0,2428 \\
\hline & $\left(e_{\mathrm{s}}\right)$ & $-0,59^{(\mathrm{ns})}$ & $-0,1652$ & $\left(e_{\mathrm{s}}\right)$ & $-1,15^{(\mathrm{ns})}$ & $-0,3279$ \\
\hline & $(\omega)$ & $-0,27^{(* * *)}$ & $-1,8706$ & $(\varphi)$ & $0,66^{(*)}$ & 2,5746 \\
\hline & $\left(\mathrm{R}^{2}\right)$ & 0,1321 & & $(\omega)$ & $-0,91^{(* *)}$ & $-2,4336$ \\
\hline & DW & 1,5901 & & $\left(\mathrm{R}^{2}\right)$ & 0,2502 & \\
\hline & & & & $\mathrm{ARCH}$ & 0,0603 & $0,970^{\text {(p.v) }}$ \\
\hline \multirow[t]{6}{*}{ BRA / EUA - Países Baixos } & Cte & $0.08^{(\mathrm{ns})}$ & 0.6162 & Cte & $-0.02^{(\mathrm{ns})}$ & -0.2923 \\
\hline & $\left(e_{\mathrm{s}}\right)$ & $-3.63^{(* *)}$ & -2.6324 & $\left(e_{\mathrm{s}}\right)$ & $-3.37^{(*)}$ & -5.5970 \\
\hline & $(\omega)$ & $-0.43^{(* *)}$ & -2.2487 & $(\varphi)$ & $1.00^{(*)}$ & 10.504 \\
\hline & $\left(\mathrm{R}^{2}\right)$ & 0,3263 & & $(\omega)$ & $-1.04^{(*)}$ & -4.1394 \\
\hline & DW & 1,9907 & & $\left(\mathrm{R}^{2}\right)$ & 0,8747 & \\
\hline & & & & $\mathrm{ARCH}$ & 0,1561 & $0,924^{(p . v)}$ \\
\hline
\end{tabular}

Notas: (1) O modelo 2 difere do modelo 1 por incluir a variável dependente defasada como variável explicativa. (***) Significativo a 10\%, (**) significativo a $5 \%,(*)$ significativo a $1 \%$, e (ns) não-significativo. (p.v) $P$-valor do teste de autocorrelação. Fonte: Resultados da pesquisa. 
Tanto o modelo 1 como o modelo 2 mostram que, entre os países importadores de soja em grão, o que apresenta maior resposta a preços é a Espanha. Os coeficientes estimados para a elasticidade de substituição foram de -5,75 e -6,09, quando o Brasil competia com a Argentina, e de $-7,35$ e $-5,12$, quando o Brasil competia com os EUA, respectivamente nos modelos 1 e 2. Esses valores elevados ${ }^{7}$ indicam que a Espanha é sensível a variações nos preços, tendo, conseqüentemente, maior suscetibilidade em substituir tanto o produto argentino quanto o norteamericano pelo produto de origem brasileira.

A Alemanha apresentou maior rigidez de mercado ${ }^{8}$, associada à menor resposta a variações nos preços da soja em grão, em comparação com a Espanha. Entretanto, também apresentou sensibilidade a preços, com coeficiente estimado de $-4,45$.

$\mathrm{Na}$ França ocorreu ajustamento defasado positivo, além de respostas também significativas à elasticidade de substituição da soja em grão do Brasil, em relação aos EUA. Isso indica a ocorrência de dois resultados desejáveis: a) A melhor solução, que seria a obtenção de um coeficiente de substituição maior que a unidade, em módulo; e b) Uma vez impossível de realizar a substituição do produto concorrente, que o país seja, no mínimo, capaz de manter sua parcela de mercado.

\footnotetext{
${ }^{7}$ Tourinho et al. (2003) classificaram as elasticidades de substituição como segue: a) muito alta, para valores acima de 3; b) alta, para valores de 1,5 a 3; c) média, para valores de 0,5 a 1,5; e d) baixa, para valores menores que 0,5.

${ }^{8}$ A rigidez de mercado é calculada pela inclusão da variável dependente defasada, como variável explicativa, no modelo de elasticidade substituição - neste caso, as quantidades relativas de exportação do Brasil e dos EUA. Este parâmetro é incluído no modelo sob a pressuposição de existência de fatores que possam limitar a substituição do produto norte-americano pelo produto brasileiro quando há alterações nos preços relativos. Dentre esses fatores destacam-se as preferências de cada mercado consumidor/importador, a tradição nas relações comerciais entre país importador e exportador, a intervenção governamental, os acordos bilaterais etc. Quando o coeficiente de rigidez de mercado se apresentar com sinal positivo, significa que a quantidade relativa exportada no período (t) será influenciada positivamente pela quantidade relativa exportada no período (t-1); assim, há evidências de que esses fatores atuam, de forma favorável, na colocação do produto brasileiro no mercado importador analisado e vice-versa, quando o sinal dessa estimativa se apresentar negativo. Por isso, um sinal positivo para a rigidez de mercado, associado a uma elasticidade de substituição baixa, significa que há evidências de diferenciação por origem na comercialização deste produto.
} 
O Japão apresentou alta rigidez de mercado, com coeficiente positivo de 1,07. Esse resultado sugere que há intervenção governamental no mercado importador japonês, porém o sinal positivo indica que o Brasil pode manter sua participação nesse mercado, com certa dificuldade em fechar novos contratos. Figueiredo (2004) destacou que as exportações brasileiras têm conseguido pequena penetração nesse mercado.

Nos Países Baixos, os coeficientes de elasticidade de substituição foram de -3,63 e -3,37, respectivamente nos modelos 1 e 2, para competição entre o Brasil e os EUA. Ademais, o coeficiente da rigidez de mercado foi positivo e igual a 1,00, o que significa que, além existir possibilidade de substituir o produto do concorrente, o Brasil tem condições de manter sua participação nas importações desse mercado. Não foi possível fazer inferências, a partir dos resultados, sobre esse mercado, estabelecendo concorrência de preços entre Brasil e Argentina.

A combinação de resultados da elasticidade de substituição e da rigidez de mercado, na França e nos Países Baixos, sugere que há alguma diferenciação por origem nas importações destes, favoráveis ao produto brasileiro. Evidência disso está na variação da parcela média do Brasil nas importações de soja em grão da França - de 16,31\% para 34,79\% e nas importações dos Países Baixos - de 30,96\% para 52,76\%, respectivamente do subperíodo 1990/96 para 1997/02 (Figueiredo, 2004). Enquanto isso, parece haver diferenciação por origem nas importações do Japão em relação ao produto dos EUA, o que implica dificuldades de os concorrentes adquirirem parcela significativa desse mercado.

\section{Conclusões}

Os valores estimados para elasticidade de substituição mostraram que, apesar de ser tratada como commodity, não há substitutibilidade perfeita entre as exportações de soja em grão do Brasil, dos EUA e da Argentina. Isso sugere possível competição de preços nesse mercado; assim, é importante que o Brasil continue buscando redução de custos, que é hoje 
um dos caminhos para a maior competitividade.

No entanto, em alguns mercados analisados, a combinação dos resultados da elasticidade de substituição com rigidez de mercado indica diferenciação por origem nas exportações. Essa diferenciação pode ser explicada por fatores culturais, acordos preferenciais, facilidades na obtenção de crédito, preferências do mercado importador associadas à origem e à qualidade do produto, plantio de sementes geneticamente modificadas, entre outros. Dessa forma, investimento em tecnologia, seja para criação de novos produtos à base de soja, seja para diferenciação do produto via rastreabilidade e preservação de identidade, é também um caminho viável ao aumento das exportações no médio e longo prazo.

\section{Referências bibliográficas}

ARMINGTON, P.S. A theory of demand for products distinguished by place of production. International Monetary Fund Staff Papers, v. 26, p. 159-178, 1969a.

ARMINGTON, P.S. The geographic pattern of trade and the effects of price changes. International Monetary Fund Staff Papers, v. 16, p. 179-199, 1969b.

BACCHI, M.R.P., BARROS, G. S. C., BURNQUIST, H. L. Estimação de equações de oferta de exportação de produtos agropecuários para o Brasil (1992/2000): Rio de Janeiro: IPEA, 2002. [25 maio 2003]. (http://www.ipea.gov.br). (Texto para discussão n. 865).

BINGER, B.R.; HOFFMAN, E. Microeconomics with calculus. 2. ed. Massachusetts: Addison-Wesley, 1998. 633 p.

FIGUEIREDO, A.M. Diferenciação por origem na competitividade das exportações mundiais da agroindústria de soja, no período de 1990 a 2002. Viçosa, MG: UFV, 2004. 133 p. Tese (Mestrado em Economia Aplicada) - Universidade Federal de Viçosa, 2004. 
FIGUEIREDO, A.M., SILVA, T.A. Exportação brasileira de soja em grãos: evolução e considerações sobre seus determinantes para o período de 1980-2001. Organizações Rurais e Agroindustriais, Lavras, v. 6, n. 1, jan./jun. 2004.

FONTES, R.M.O, GREEN, T., JOHNSON, P. Diferenciação de produtos e ajustamento defasados numa análise das exportações de grãos dos Estados Unidos. Revista de Econometria, Rio de Janeiro, v. IX, n. 2, p. 211-29, 1989.

FOOD AND AGRICULTURE ORGANIZATION OF THE UNITED NATIONS - FAO. FAOSTAT Database. [20 jan. 2004]. (http:// apps.fao.org/).

GRIFFITHS, W., HILL, C., JUDGE, G. Econometria. São Paulo: Saraiva, 2000. $408 \mathrm{p}$.

GUJARATI, D. N. Econometria básica. 3. ed. São Paulo: Makron Books, 2000. $846 \mathrm{p}$.

LEAMER, E.E., STERN, R.M. Quantitative international economics. Chicago: Aldine Publ., 1970. p. 171-183.

MINISTÉRIO DA AGRICULTURA, INDÚSTRIA E COMÉRCIO EXTERIOR - MDIC. Estatísticas de exportação e importação brasileira 1989 a 2004. [20 jan. 2004]. (http:// aliceweb.desenvolvimento.gov.br/).

MINISTÉRIO DE ECONOMIA Y PRODUCCIÓN DA ARGENTINA - MECON. Estadística y censos de la Republica Argentina. [20 jan. 2004]. (www.sagpya.mecon.gov.ar).

SILVA, O.M. Elasticidade de substituição para o suco de laranja no mercado internacional. Revista de Economia e Sociologia Rural, Brasília, v. 30, n. 2, p. 136-147, abr./jun. 1992.

STALDER, S.H.G.M. Análise da participação do Brasil no merca- 
do internacional de açúcar. Piracicaba, SP: ESALQ, 1997. 121 p. Dissertação (Mestrado em Economia Aplicada) - Escola Superior de Agricultura Luiz de Queiroz, 1997.

TOURINHO, O.A.F., KUME, H., PEDROSO, A.C.S. Estimativas de armington para o Brasil - 1986-2002: novas estimativas. Rio de Janeiro: IPEA, 2003. [01 maio 2004]. (www.ie.ufrj.br/eventos/ seminarios/pesquisa/elasticidades_de_armington_para_o_brasil.pdf). (Texto para discussão n. 974).

UNITED STATES DEPARTMENT OF AGRICULTURE - USDA. Foreign trade statistics. [21 dez. 2003]. (http://www.fas.usda.gov/ ustrade/USTExFatus.asp? QI=).

VARIAN, H.R. Microeconomia: princípios básicos. Rio de Janeiro: Campus, 1999. 740 p.

VIANA, J.J.S, SILVA, O.M., LIMA, J.E., CARVALHO, F.M.A. Diferenciação por origem na demanda internacional de cafés. In: CONGRESSO BRASILEIRO DE ECONOMIA E SOCIOLOGIA RURAL, 41, 2003, Juiz de Fora. Anais... Brasília, DF: SOBER, 2003. 1 CD-ROM.

\footnotetext{
Abstract - The objective of this work was to estimate and to analyze the elasticity of substitution between Brazil, the USA and Argentina in the soybeans exports in the selected importers markets. This fact is important, considering that, it increases the integration of the world economies, placing in prominence of the adaptability to the world demand, as a fundamental factor for the exports expansion. Like this, given the economical relevance of the Brazilian exports of soybeans it was looked for, specifically, to verify the substitution possibility between the soybeans exports of Brazil, the USA and Argentina. After accomplishment of Dickey-Fuller expanded tests (ADF) for unit root, and Trace test and Max-eingevalue test for co-integration, the Error Correction Model (ECM) was adopted in the estimate of the elasticity of substitution. That model was shown well adjusted to the datas, allowing to identify the presence or absence of market rigidity, even so there is not perfect substitution in the soybeans exports. This way, investment in differentiation of the product, through rastreability and identity preservation can impulse the Brazilian exports.

Keywords: Elasticity of substitution, exports, soybeans, Brazil.
} 
REVISTA DE ECONOMIA E AGRONEGÓCIO, VOL.2, $N^{\circ} 4$ 